“ (C) 2017 IEEE. Personal use of this material is permitted. Permission from IEEE must be obtained for all other uses, in any current or future media, including

reprinting/republishing this material for advertising or promotional purposes, creating new collective works, for resale or redistribution to servers or lists, or reuse of any copyrighted component of this work in other works." 


\section{Performance Comparisons between Force-directed Algorithms on Structured Data Analysis}

\author{
Jie Hua \\ University of Technology Sydney \\ Sydney, Australia \\ e-mail: Jie.hua@alumni.uts.edu.au
}

\author{
Mao Lin Huang \\ University of Technology Sydney \\ Sydney, Australia \\ e-mail: Mao.Huang@uts.edu.au
}

\author{
Guohua Wang \\ South China University of Technology \\ Guangzhou, China \\ e-mail: ghwang@scut.edu.cn
}

\begin{abstract}
Force-directed algorithms have been widely applied in practical data visualization area due to their capabilities of producing good layouts, which follows metrics for graph drawing aesthetics, yet evaluation the performance of relevant algorithms is still a challenge, since layout quality is largely relying on personal judgement and/or methods' input parameters, and most aesthetics criteria conflict with each other. This study evaluates the performance measurements of four algorithms in terms of seven commonly applied aesthetic criteria and demonstrates the experimental framework.
\end{abstract}

Keywords- Graph drawing; force-directed algorithms; aesthetic criteria; evaluation

\section{INTRODUCTION}

Force-directed algorithms have been widely applied in graph drawing field. Graphs are treated as physical systems, forces (spring force, gravity force etc.) are applied to each element (vertex/edge), and the process stops when zero(minimized) energy is reached or a stop condition is determined $[3,4,5,6,7,8,9,16]$. It was first introduced based on barycentric representations (Tutte, 1963). The spring layout methods rely on spring forces, similar to those in Hooke's law (Eades, 1984; Fruchterman \& Reingold, 1991). And in those methods, there are repulsive forces between all vertices and also attractive forces between adjacent vertices. Alternatively, spring forces can be computed based on their graph theoretic distances (Kamada \& Kawai, 1989). Graphs drawn with these force-directed algorithms tend to be aesthetically pleasing, exhibit symmetries, and most likely to produce crossing-free layouts for planar graphs [10].

Force-directed algorithms can generate different layouts of the same dataset, depending on the combination of input parameters such as initial placement of vertex's and constants that define the physical forces schedule [10]. All these lead to a challenge to compare performance among those common applied force-directed algorithms. And achieving a better layout of a particular graph remains unclear.

In this paper, a case study was conducted based on practical raw data collected from the Australian stock market, to compare four force-directed approaches, in terms of seven aesthetic criteria in graph drawing.

\section{RELATED WORK}

Brandenburg, Himsolt and Rohrer compared five forcedirected algorithms for general undirected graphs, following measurements factors such as running time, edge length ratio/standard deviation and edge crossing etc. Yet, less empirical evidence was concluded [12].

Four general-purpose graph drawing methods were compared by Battista et al. and edge crossing/length/bends etc. were evaluated in testing, but the force-directed approach was not involved in detailed experiments [13].

Gansner and North presented the application of two postprocessing techniques to produce uncluttered layouts with non-point nodes, to improve readability in labelled nodes for force-directed models [14]. The comparisons were limited between the Voronoi and scaling though.

Hachul and Jünger investigated several methods such as GVA, HDE and $\mathrm{FM}^{3}$ etc. for large graph drawing, addressing the time complexity, however, criteria for layout performance judgment were 'pleasing' feature [15].

Huang, Eades, Hong, and Lin argued that effectiveness could be improved when algorithms are designed by making compromises between aesthetics, rather than trying to satisfy one or two of them to the fullest. Their study indicates that BIGANGLE induces significantly better performance of humans in perceiving shortest paths between two nodes [2].

The performance comparisons of two different forcedirected algorithms in terms of six commonly applied aesthetic criteria were evaluated by Huang and Lin. The results indicate that not only the aesthetics that are supposed to be improved by the algorithm but also other aesthetics that are important to the overall visual quality of drawings [1].

However, performance on final layouts from methods above were mainly judged based on personal opinion, and/or it is largely relying on methods' input parameters such as initial layouts etc. $[1,11]$, which lacks empirical evidence; in addition, there are conflictions between most of the aesthetics criteria related to layout quality, thus conducting evaluation on algorithm performance measurement has become a challenging issue in graph drawing area. 


\section{EVALUATION FRAMEWORK}

The evaluation framework is proposed as follows:

- Stock raw data collection from Australian Securities Exchange (ASX);

- Graph model conduction, it involves data preparation (data filtering/cleansing/formatting etc.), data processing, and crossing-comparisons on cleaned data based on close vales of each stock data, sees [17] for data processing details. Eventually, five undirected/weighted graphs were built up.

- Force-directed algorithms selection;

Four common force-directed algorithms applied for evaluation.

- Graph layouts generation;

Applying selected force-directed algorithms on graph models created.

- Measure and compare the aesthetic quality of the resultant drawings based on seven factors, hence, to find out the better approach that suits generalpurposed aesthetic criteria requirements in graph drawing fields.

\section{CASE STUDY}

The evaluation framework is proposed as follows:

\section{A. Force-directed Algorithms}

Four algorithms were adopted in evaluation framework: Fruchterman and Reingold (FR), ForceAtlas (FA), ForceAtlas2 (FA2) and Linlog. Initial layouts for graph models were produced randomly.

Fruchterman and Reingold proposed an algorithm follows two principles as 1) Vertices connected by an edge should be drawn near each other. 2) Vertices should not be drawn too close to each other. To produce aestheticallypleasing, two-dimensional pictures of graphs by doing simplified simulations of physical systems [3]. The method is simple, elegant, conceptually intuitive, and efficient, comes with uniform edge lengths.

FA aims at giving a readable shape to a network (spatialization), along with integration between different techniques such as Barnes Hut simulation, degree-dependent repulsive force, local and global adaptive temperatures. It addresses providing a generic and intuitive way to spatialize networks. FA2 is based on FA but offers more options and innovative optimizations that make it a very fast layout algorithm. Its implementation of adaptive local and global speeds brings good performances for a network of fewer than 100000 nodes. It was empirically observed that FA2 is at its best with strongly clustered networks. FA2's ability to show clusters is better than FR algorithm and worse than Linlog's [16].

Noack proposed the Linlog energy models, including node-repulsion Linlog and edge-repulsion Linlog, whose minimum energy layouts reflect the cluster structure of graphs with respect to two well-defined clustering criteria, and edge repulsion in energy models, avoids or reduces the bias towards grouping nodes with high degree when used instead of or in addition to node repulsion [8].

\section{B. Graph Models}

In experimental evaluation:

- raw data were collected from the ASX, including 5088 stocks in Australia, ranges from 02/01/1997 to 30/06/2017, around 6.4 million data entries (before formatted/filtered);

- after cross comparison on related changing rates between every two stocks, nearly 194 million raw data were generated;

- after data cleansing step, five groups of structured data which followed different filtering rules have been kept for testing;

- five connected/undirected/weighted graphs were finalized artificially based on cleansed raw data, to test the proposed framework then.

Five graph models are:

- $G_{l}=\left(V_{l}, E_{l}\right)\left(\left|V_{l}\right|=115,\left|E_{l}\right|=497\right)$;

- $G_{2}=\left(V_{2}, E_{2}\right)\left(\left|V_{2}\right|=252,\left|E_{2}\right|=1668\right)$;

- $G_{3}=\left(V_{3}, E_{3}\right)\left(\left|V_{3}\right|=317,\left|E_{3}\right|=3572\right)$;

- $G_{4}=\left(V_{4}, E_{4}\right)\left(\left|V_{4}\right|=334,\left|E_{4}\right|=6654\right)$

- $G_{5}=\left(V_{5}, E_{5}\right)\left(\left|V_{5}\right|=339,\left|E_{5}\right|=11473\right)$;

\section{Aesthetic Creieria}

Battista, Eades, Tamassia and Tollis claimed that there were several common aesthetics for general undirected graph drawing, which includes: symmetry display; edge crossing reduction; edge bends reduction, edge length uniform and vertex distribution uniform etc. [4].

Finkel and Tamassia compared angular resolution and edge separation as relevant aesthetic criteria. The angular resolution refers to the angles formed by pairs of edges incident on a vertex. The edge separation refers to the distance between an edge and another non-incident, nonintersecting edge [6].

Huang indicated that human graph reading performance can be affected by the size of crossing angles. The maximizing the size of crossing angles has been shown beneficial for graph comprehension [11]. And average size of crossing angles (angle size), Standard deviation of crossing angle (angle dev.) etc. were adopted in experiments, see [1], [2] and [11].

In our experiments, the following aesthetic criteria were applied for algorithm performance measurement:

- Cross\#: Edge Crossing Number.

The number of edge crossings should be minimized whenever possible in drawing graphs [5].

- Angle Size: Average size of crossing angles. The crossing angle criterion that maximizing crossing angles can make graph drawings more readable [11]. In our testing, suppose $\theta$ is the original angle size, the finalized angle size is represented using $\left(90^{\circ}-\theta\right)$, thus, the smaller the finalized angle size is, the better to final layout.

- Angle Dev.: The standard deviation of crossing angles. 
A smaller difference implies a better angel dev.

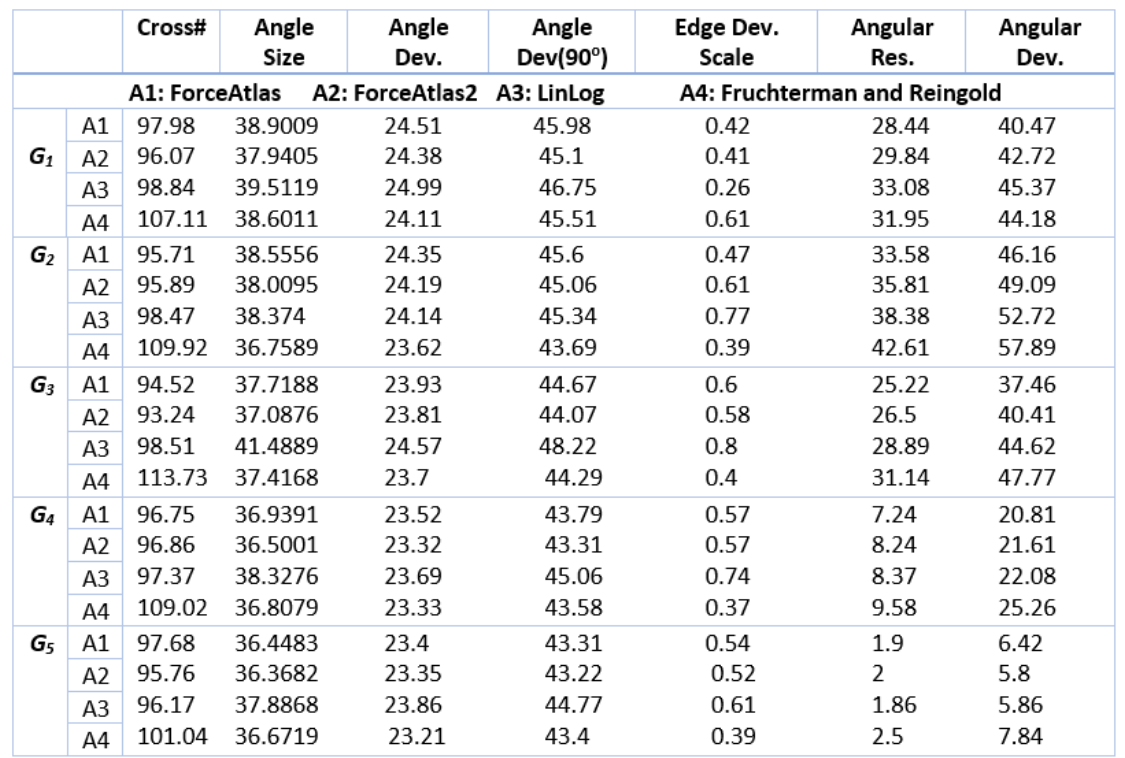

Figure 1. Experiments results

- Angle Dev. $\left(90^{\circ}\right)$ : The standard deviation of difference on crossing angles to $90^{\circ}$.

A smaller difference implies a nearer angle to $90^{\circ}$ and easier-to-recognize crossing.

- Edge Dev. Scale: EdgeDev./EdgeLength (Suppose EdgeDev. Means standard deviation of edge length, and EdgeLength indicates average edge length).

A smaller difference implies a better-uniformed edge length. One of the main aesthetics in graph drawing is to keep edge lengths uniform [4].

- Angular Res.: Angular resolution is measured as the average of differences between the smallest angle and the optimal angle for each vertex.

A smaller difference implies a better angular resolution (a better layout) [6] (Suppose that vertex $a$ has at least two incident edges. Let $\varphi$ be the optimal angle $\left(360^{\circ} / \operatorname{deg}(a)\right), \theta$ be the angle formed by a pair of two neighboring edges $(a, b)$ and $(a, c))$.

- Angular Dev.: The standard deviation of angular res. A smaller difference implies a better angular deviation.

\section{Experimental Results}

We applied four force-directed algorithms on five graph models generated, and compare the final layouts based on seven performance measurements described above (edge crossing number and angle size etc.).

Fig. 1 shows the final testing results. For example, the edge crossing number on $G_{l}$ 's final layout after applied FA is $97.98 * 10 \mathrm{k}$, and it is $107.11 * 10 \mathrm{k}$ after applied FR, which means FA is 'better' than FR in this simple case.

From Fig. 1 that in $G_{1}$, between FA2 and FR, FA2 produces $10.3 \%$ less edge crossing and $6.7 \%$ less angular resolution; In $G_{4}$, it presents $5.2 \%$ less edge crossing, $0.8 \%$ less average angle size and $20 \%$ less angular resolution as well. In most cases from testing, FA2 produced better layouts which kept balance in those aesthetic criteria factors; FR has the worst edge crossing.

The final comparisons are shown in Fig. 2. It offers a parallel coordinates layout in which five colour groups indicate five different graph models, and the five highlighted blue lines present aesthetic measurements of final layouts from the FA2 method. Scale adjustments have been done on final testing data to avoid group overlap, to offer a 'clear' view for measurement. Fig. 2's results also support that FA2 satisfies aesthetic criteria in graph drawing most, it excels in edge crossing reduction, angle size maximization and angle $\mathrm{dev} . / \mathrm{dev} .\left(90^{\circ}\right)$, and conducts layouts well in edge dev.scale, angular res./dev. FR method can offer more uniformed edge length. In addition, the results show that layouts come with less angular resolution/deviation tends to have less edge crossing.

\section{CONCLUSIONS AND FUTURE WORKS}

Based on practical structured data collected and finalized, our experiments compared four common force-directed algorithms, following seven aesthetics criteria, the early outcomes show that FA2 provides above 'average' performance layouts, come with less edge crossing and angular resolution etc. Those experimental results offer detailed measurements with empirical evidence other than only personal judgement. In our experiments, only highly structured data were involved, and several aesthetics factors have been measured, which may affect the final accuracy of experiment outcomes. And since most graph drawing aesthetics criteria conflict with each other, detailed forcedirect algorithm selection is still relying on specific requirements. In our future work, more factors such as time complexity and more data types will be considered. 


\section{REFERENCES}

[1] M. Huang, W. Huang, and C. Lin, "Evaluating Force-Directed Algorithms with a New Framework," in the 27th ACM Symposium on Applied Computing (SAC'12), 2012, pp. 1030-1032.

[2] W. Huang, P. Eades, S. H. Hong, and C. C. Lin, "Improving forcedirected graph drawings by making compromises between aesthetics," Proc. - 2010 IEEE Symp. Vis. Lang. Human-Centric Compute. VL/HCC 2010, pp. 176-183, 2010.

[3] T. M. J. Fruchterman and E. M. Reingold, "Graph drawing by forcedirected placement," Software Practice and Experience., vol. 21, no. 11, pp. 1129-1164, 1991.

[4] G. Di Battista, P. Eades, R. Tamassia, and I. G. Tollis, "Algorithms for Drawing Graphs an Annotated Bibliography," Comput. Geom. Theory Appl., vol. 4, pp. 235-282, 1994.

[5] G. Di Battista, P. Eades, R. Tamassia, and I. G. Tollis, Graph drawing: algorithms for the visualization of graphs. 1999.

[6] B. Finkel and R. Tamassia, "Curvilinear Graph Drawing Using the Force-Directed Method," in the 12th International Symposium on Graph Drawing (GD’04), 2004, pp. 448-453.

[7] M. L. HUANG, P. EADES, and J. WANG, "On-line Animated Visualization of Huge Graphs using a Modified Spring Algorithm," J. Vis. Lang. Comput., vol. 9, no. 6, pp. 623-645, 1998.

[8] A.Noack, "Energy Models for Graph Clustering," J. Graph Algorithms Appl. JGAA, vol. 11, no. 112, pp. 453-480, 2007.

[9] Y. Hu, "Efficient and High Quality Force-Directed Graph Drawing," Math. J., vol. 10, no. 1, pp. 37-71, 2005.
[10] S. G. Kobourov, "Force-directed drawing algorithms," Handb. Graph Draw. Vis. (Discrete Math. Its Appl., pp. 383-408, 2013.

[11] W. Huang and M. Huang, "Exploring the Relative Importance of Number of Edge Crossings and Size of Crossing Angles: A Quantitative Perspective,” Int. J. Adv. Intell., vol. 3, no. 1, pp. 25-42, 2011.

[12] F.J. Brandenburg, M. Himsolt, and C. Rohrer, "An experimental comparison of force-directed and randomized graph drawing algorithms," Proc. Symp. Graph Draw. (GD 1995), pp. 76-87, 1996.

[13] G. Di Battista, A. Garg, G. Liotta, R. Tamassia, E. Tassinari, and F. Vargiu, "An experimental comparison of four graph drawing algorithms," Comput. Geom., vol. 7, no. 5-6, pp. 303-325, 1997.

[14] E. R. Gansner and S. C. North, "Improved force-directed layouts," in Proceedings of Graph Drawing 98, 1998, pp. 364-373.

[15] S. Hachul and M. Jünge, "An Experimental Comparison of Fast Algorithms for Drawing General Large Graphs," in Proc. 13th Int'l Symp. Graph Drawing (GD ’05), 2005, pp. 235-250.

[16] M. Jacomy, S. Heymann, T. Venturini, and M. Bastian, "Force Atlas 2, a graph layout algorithm for handy network visualization," .. Http//www. Medialab. Sci. Fr/ ..., pp. 1-21, 2011.

[17] M. Zreika, J. Hua, and G.H. Wang, "Applying Data Processing Method for Relationship Discovery in the Stock Market," in the International Conference on Data Science and Business Analytics (ICDSBA 2017), 2017, unpublished.

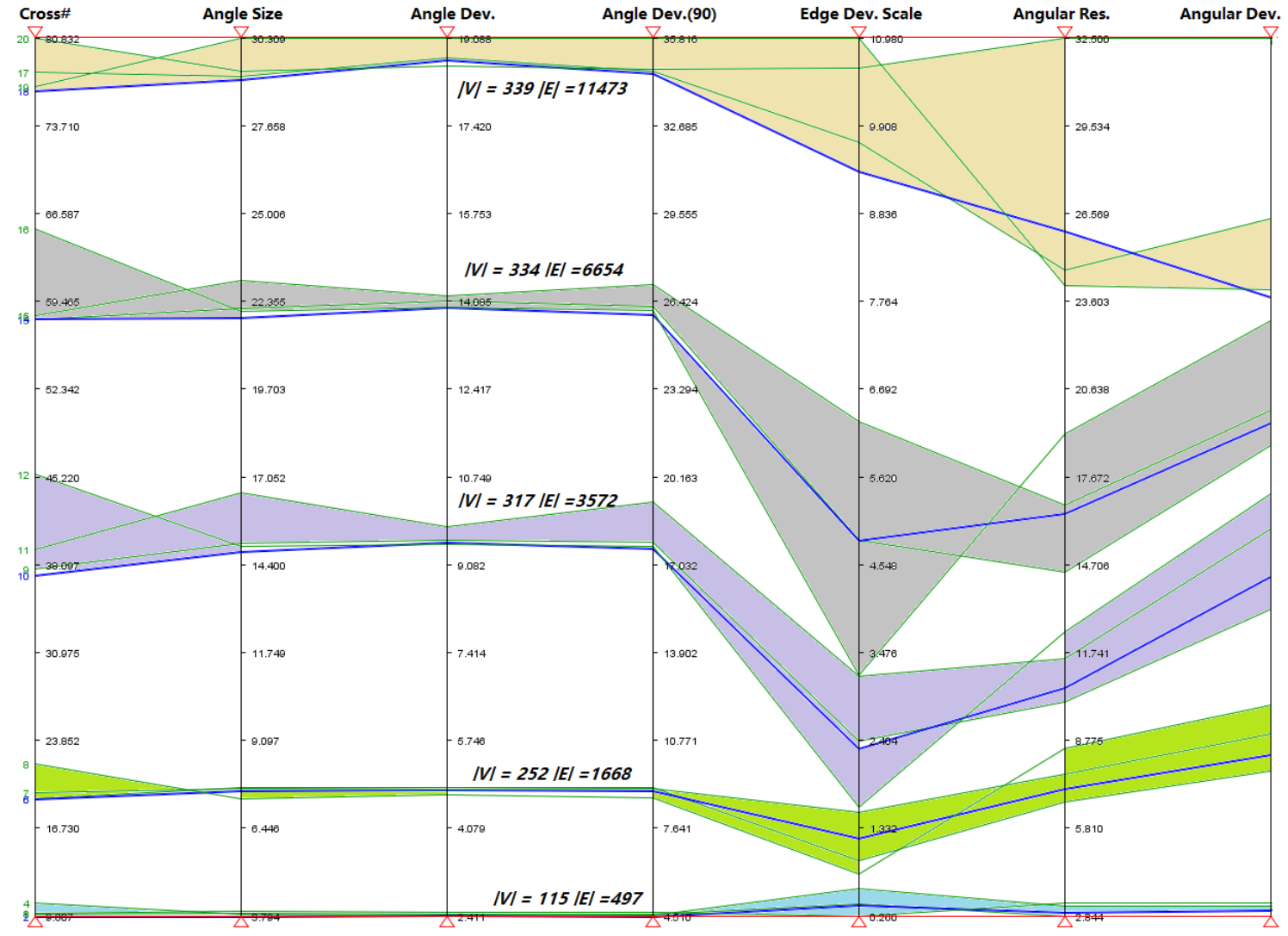

Figure 2. Comparisons of aesthetic measures 